\title{
Integración de la lógica difusa a la dinámica de sistemas para la selección de terrenos de cultivos agrícolas
}

\author{
Integration of Fuzzy Logic with System \\ Dynamics for the Selection of Farming Soils
}

\author{
Natalia Martínez Rojas ${ }^{\star}$, Hugo Hernando Andrade Sosa ${ }^{\star \star}$ \\ Universidad Industrial de Santander
}

FeCha De Entrega: 14 DE ABRIL DE 2016

FECHA DE EVALUACIÓN: 10 DE MAYO DE 2016

FECHA DE APROBACIÓN: 27 DE MAYO DE 2016

\begin{abstract}
Resumen La instalación de un sistema agrícola es un proceso complejo que requiere de un conocimiento tanto del tipo de cultivo como de análisis de terrenos de tal forma que se puedan obtener los mayores beneficios. Es por esto que el propósito de este artículo es integrar la lógica difusa a modelos realizados con Dinámica de Sistemas, con el fin de apoyar la toma de decisiones de los agricultores. Con el modelo, el productor puede simular y experimentar de forma iterativa para evaluar posibles escenarios que le permitan tomar acciones correctivas antes de realizar la instalación de determinado cultivo.
\end{abstract}

\begin{abstract}
The installation of a farming system is a complex process that requires knowing the type of crop and the analysis of the soil to obtain more benefits. For this reason, the purpose of this article is to integrate fuzzy logic with models made with system dynamics in order to support the farmers on the decision making process. With this model, the producer can simulate and repeatedly experiment the evaluation of possible scenarios that enable corrective actions before growing a specific crop.
\end{abstract}

Palabras Clave: Dinámica de Sistemas, lógica difusa, suelos, agricultura, toma de decisiones.

Keywords: System dynamics, fuzzy logic, soil, agriculture, decision making.

\footnotetext{
* natica@simon.uis.edu.co

${ }^{\star \star}$ handrade@uis.edu.co
} 


\section{Introducción}

Este artículo muestra cómo, resultado de integrar la lógica difusa con la Dinámica de Sistemas, surge un lenguaje de construcción y reconstrucción de conocimiento que al poder considerar raciocinios de carácter cuantitativo y cualitativo, junto con las potencialidades de la representación de sistemas realimentados fundados en ecuaciones diferenciales lineales y no lineales; permite abordar la complejidad dinámico sistémica de los asuntos en los cuales el ser humano toma de decisiones con propósito de intervención [6].

El modelado y la simulación por computador permiten apoyar y facilitar la evaluación de un conjunto de parámetros para el estudio y análisis de terrenos. Ellos proveen por medio de herramientas tecnológicas, la información necesaria, para que el agricultor o profesional pueda tomar decisiones en función de las metas

de producción. Estas herramientas, son concebidas como sistemas expertos que apoyan la toma de decisiones [10]; donde el usuario analiza, interpreta y evalúa, diferentes escenarios de producción en función de la información registrada y procesada por el sistema de inferencia difuso; lo cual, conduce a maximizar la productividad [9].

El uso de la lógica difusa en la evaluación de terrenos es de particular importancia ya que es posible minimizar el efecto de cualquier característica cuando alcanza un valor inmediatamente exterior a su rango establecido, como se presenta en la lógica clásica [15] [4]. Los controladores difusos aprovechan las expresiones lingüísticas suministradas por los expertos que tienen amplio conocimiento en el estudio de los terrenos [8].

\section{Metodología}

A continuación se presenta brevemente la Dinámica de Sistemas (DS) en su proceso de modelado, la Lógica Difusa en la construcción de los sistemas de inferencia difusa integrables en el modelo de DS; para luego ilustrar las potencialidades de la integración propuesta, en términos de un modelo de simulación para cultivos agrícolas con valoración cuantitativa y cuantitativa del suelo.

\section{Dinámica de Sistemas}

Los modelos con Dinámica de Sistemas representan los flujos de material (procesos) y de información a través de los cuales se conectan los estados del sistema con los puntos de decisión, y también con las consecuencias de esas decisiones, mostrando ciclos de realimentación de refuerzo o balance. Dado que estos ciclos incluyen, frecuentemente, retardos y relaciones no lineales, se hace necesario el uso de la simulación para obtener las consecuencias temporales asociadas a estas estructuras causales complejas, de forma que los comportamientos contraintuitivos puedan ser analizados de forma rigurosa para entender a qué se deben. 
Estos modelos permiten también el diseño de nuevas políticas para el sistema, así como avanzar en la comprensión de sus posibles consecuencias [26].

"La Dinámica de Sistemas es una alternativa para dar cuenta de la complejidad dinámica de los fenómenos explicables en términos de sistemas en donde la variedad en los campos de aplicación la proponen como una metodología" [13]. La DS provee una herramienta de comunicación común conectando varias disciplinas académicas, ya que fuerza a las personas a pensar críticamente sobre los problemas debido al proceso que deben seguir. Con la Dinámica de Sistemas, se puede hacer un enlace mental entre la estructura de un sistema y el comportamiento que este produce, a través de modelos, los cuales son una parte del sistema que cambia con respecto al tiempo, que comparado con un sistema en tiempo real tiene un costo más bajo y permite un conocimiento más rápido de las condiciones que no se observan en la vida real [2].

En la literatura se pueden encontrar clasificaciones de las etapas en las que se puede realizar un estudio de Dinámica de Sistemas: Forrester [11], Schaffernicht [25], Aracil [3]; Coyle [7]; Andrade [2] entre otras.

Basándose en dichas clasificaciones y con la intención de describir cual fue la guía a seguir en el presente estudio, se consideró un estudio de Dinámica de Sistemas siguiendo lo propuesto por Andrade. La Figura 1 muestra el esquema, resaltando que el proceso completo debe ser considerado iterativo, en el sentido en que las distintas etapas pueden ser revisitadas tantas veces como se considere necesario.

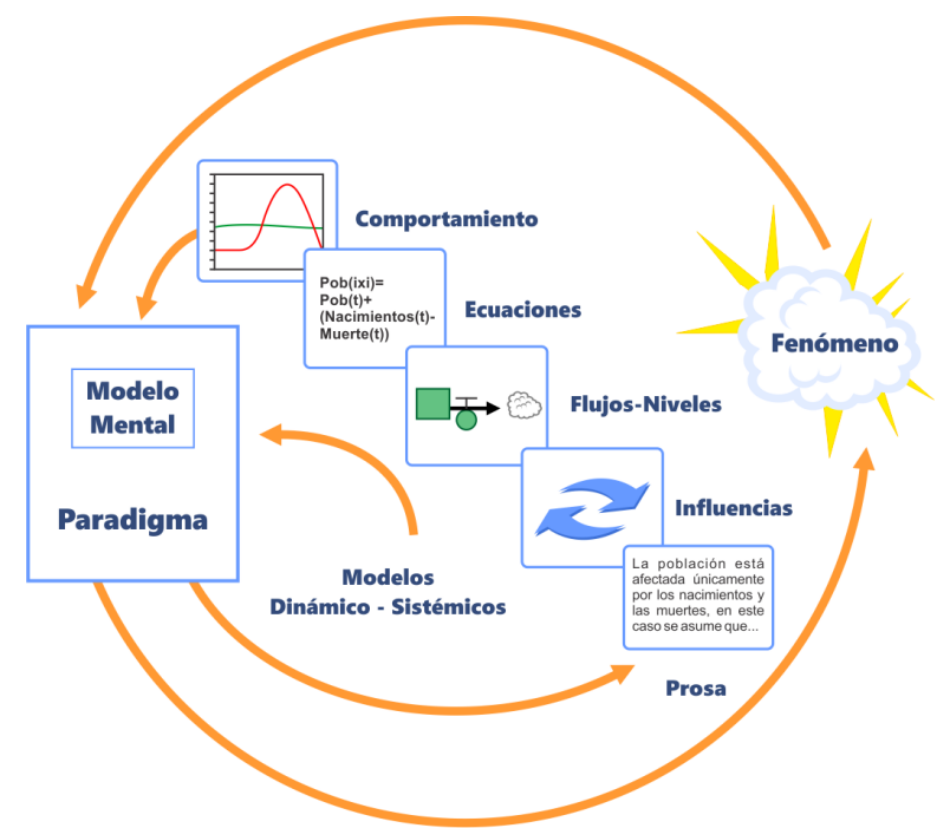

Figura 1. El modelado con Dinámica de Sistemas. Fuente: [2] 
La Dinámica de Sistemas proporciona un sistema de lenguajes con los cuales es posible expresar la causalidad. Los modelos Dinámico-Sistémicos son escritos en esos lenguajes [2]. La Figura 1 muestra los diferentes tipos de lenguajes:

1. El lenguaje en prosa, es el usado cotidianamente, es posible hablar en términos de las causalidades y como estas condicionan el comportamiento.

2. Los diagramas causales y los diagramas de flujos y niveles, tienen un carácter gráfico. En cada uno de ellos se puede dibujar y apreciar visualmente la estructura causal con sus ciclos de realimentación. Para cada uno de estos diagramas existe una lógica que permite inferir comportamientos posibles del sistema a partir de las estructuras que allí aparecen [2].

3. Las ecuaciones puede representar la estructura causal como un sistema de ecuaciones diferenciales. Con la representación matemática del sistema y haciendo uso de herramientas especializadas, se puede simular en el computador, con amplias facilidades para la interacción en un lenguaje visual de definición de escenarios de simulación y presentación de comportamientos [2]. Al aporte de la dinámica de cambio que hacen las ecuaciones diferenciales, es posible integrar aportes de otras herramientas matemáticas como la lógica difusa, las redes neuronales, los algoritmos genéticos y la estadística en representaciones de sucesos determinísticos o estocásticos.

Cada uno de los lenguajes de la Dinámica de Sistemas satisface de manera particular los requerimientos de un modelo Dinámico-Sistémico y en conjunto las representaciones dinámico sistémicas sustentan y presentan un modelo de Dinámica de Sistemas, aunque una representación en particular no necesariamente requiere de los cinco lenguajes, todo depende del propósito del modelo.

\subsection{Lógica difusa}

Es la lógica que utiliza expresiones que no son ni totalmente ciertas ni completamente falsas [19], es decir, es la lógica aplicada a conceptos que pueden tomar un valor cualquiera de veracidad dentro de un conjunto de valores que oscilan entre dos extremos, la verdad absoluta y la falsedad total.

Cabe recalcar que lo difuso, borroso, impreciso o vago no es la lógica en sí, sino el objeto que estudia, la medición y valoración de sus características. La Lógica Difusa permite tratar información imprecisa, como estatura media o temperatura baja, en términos de conjuntos difusos que se combinan en reglas para definir acciones: si la temperatura es alta entonces enfriar mucho. De esta manera, los sistemas de control basados en lógica difusa combinan variables de entrada, definidas en términos de conjuntos difusos, por medio de grupos de reglas que producen uno o varios valores de salida.

Los conjuntos difusos pueden ser considerados como una generalización de los conjuntos clásicos; la teoría clásica de conjuntos sólo contempla la pertenencia o no pertenencia de un elemento a un conjunto, sin embargo la lógica difusa considera que un conjunto no tiene una frontera clara que permita decir si un elemento pertenece o no a él. Mediante una función se asigna a cada elemento 
del conjunto un grado de pertenencia entre 0 y 1 . Este grado de pertenencia se define mediante la función característica asociada al conjunto difuso.

La única condición que debe cumplir una función característica es que tome valores entre 0 y 1 , con continuidad. Conceptualmente existen dos aproximaciones para determinar la función característica asociada a un conjunto: la primera aproximación está basada en el conocimiento humano de los expertos, y la segunda aproximación es utilizar una colección de datos para diseñar la función [20].

\begin{tabular}{|c|c|}
\hline Concepto & Descripción \\
\hline Variable lingüística & $\begin{array}{l}\text { Es aquella noción o con- } \\
\text { cepto que vamos a calificar Altura } \\
\text { de forma difusa }\end{array}$ \\
\hline Universo de discurso & $\begin{array}{l}\text { Es el rango de valores que } \\
\text { pueden tomar los elemen- } \\
\text { tos que poseen la propie- } \text { Conjunto de valores comprendido } \\
\text { dad expresada por la va- } \\
\text { riable lingüística }\end{array}$ \\
\hline Valor lingüístico & $\begin{array}{l}\text { Son las diferentes clasifica- } \\
\text { ciones que efectuamos so- "Bajo", "Mediano" y "Alto". } \\
\text { bre la variable lingüística }\end{array}$ \\
\hline $\begin{array}{l}\text { Función de pertenen- } \\
\text { cia }\end{array}$ & $\begin{array}{l}\text { Aplicación que asocia a ca- } \\
\text { da elemento de un conjun- } \\
\text { to difuso el grado con que } \\
\text { pertenece al valor lingüís- } \\
\text { tico asociado }\end{array}$ \\
\hline
\end{tabular}

Cuadro 1. Conceptos básicos de la lógica difusa

\subsection{Sistemas basados en lógica difusa}

Tradicionalmente los sistemas de control inteligente combinan la teoría clásica de control con técnicas de inteligencia artificial [23] [20]. Los sistemas de control inteligentes de mayor implantación son los controladores difusos [24].

Para la construcción de un sistema basado en reglas difusas como se muestra en la Figura 2 está compuesto por la interfaz de fuzzificación, mecanismo de inferencia, base de reglas, base de datos e interfaz de defuzzificación [16] ([4]. Esto se puede implementar en un modelo con Dinámica de Sistemas, utilizando en software Evolución, en donde un modelo cuantitativo a través del componente FIS [17] contempla consideraciones cualitativas. 


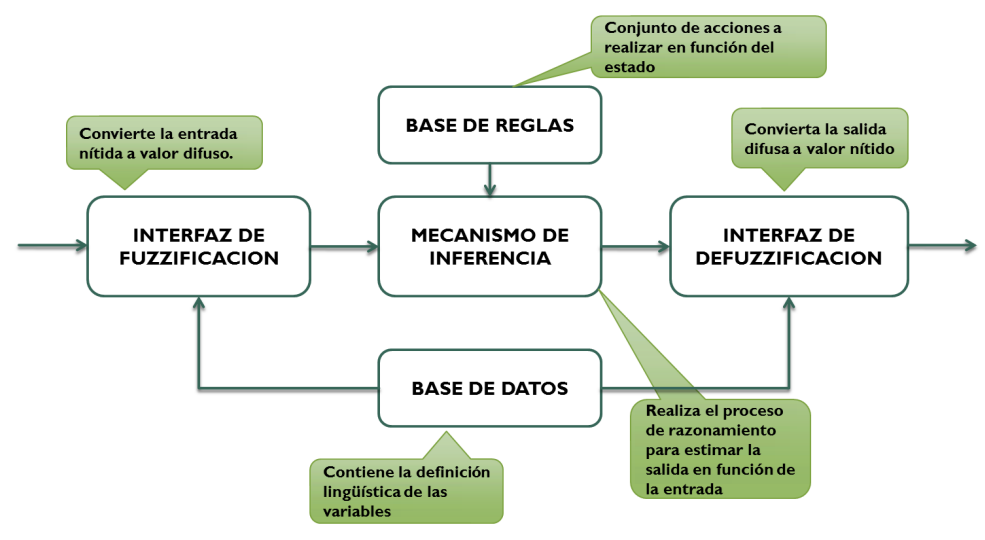

Figura 2. Estructura básica de un sistema de inferencia difuso. Fuente: Adaptación de [14]

1. Base de conocimiento

- Base de reglas: Una regla difusa if-then, es una regla del tipo si $x$ es $A$ entonces y es $B$. En donde A y B son etiquetas lingüísticas definidas en sus respectivos universos $\mathrm{X}$ e $\mathrm{Y}$.

La parte si $x$ es $A$ es el antecedente, $y$ es $B$ el consecuente.

- Base de datos: contiene la definición lingüística de las variables.

2. Mecanismo de inferencia. Cada regla es interpretada como una implicación difusa, relaciona los conjuntos de entrada y salida para representar las reglas que definen el sistema, utilizando la información de la base de conocimiento para generar reglas mediante el uso de condiciones. Consiste en tres componentes conceptuales:

- Reglas difusas

- Diccionario (con funciones de pertenencia)

- Mecanismo de raciocinio

Además en sistemas con entradas y/o salidas nítidas:

3. Interfaz de fuzzificación: Toma valores numéricos provenientes del exterior y los convierte en valores "difusos" que pueden ser procesados por el mecanismo de inferencia. Dentro de este proceso se asignan grados de pertenencia a cada una de las variables de entrada con relación a los conjuntos difusos. Estos valores difusos son los niveles de pertenencia de los valores de entrada a los diferentes conjuntos difusos en los cuales se ha dividido el universo de discurso de las diferentes variables de entrada al sistema.

4. Interfaz de defuzzificación: Realiza el proceso de adecuar los valores difusos generados en la inferencia de valores utilizados en el proceso de control. Realización de métodos matemáticos simples mediante los cuales obtenemos un valor concreto de la variable de salida. 


\section{Modelo con Dinámica de Sistemas para cultivos agrícolas con análisis y selección de terrenos}

Cuando se toma una decisión sobre cultivar o no en determinado lugar, se hace en función del resultado esperado con el paso del tiempo; resultado debido a la relación de un conjunto de parámetros y variables que se han identificado como determinantes del devenir del cultivo. Identificar los parámetros y variables del modelo y relacionarlos en términos de un sistema realimentado, así como como describir las relaciones de influencia entre los elementos del sistema en términos de expresiones matemáticas que representan a su vez raciocinios de carácter cuantitativos y de carácter cualitativo corresponde a una compleja tarea que permite el abordaje de la complejidad del fenómeno, en este caso del cultivo como se ilustra a continuación.

\subsection{Conceptualización Dinámico-Sistémica}

Generalmente el momento inicial del modelado con Dinámica de Sistemas corresponde a la etapa de conceptualización Dinámico-Sistémica del fenómeno de interés, en la cual se propone una representación básica de la estructura de realimentación que determina su dinámica. Dicha estructura de realimentación se despliega en el tiempo a la manera de un espiral y constituye la hipótesis dinámica que habrá de verificarse en la medida que todo el proceso de modelado permita explicar de manera satisfactoria el fenómeno en estudio.

La hipótesis dinámica es una representación del sistema productivo agrícola donde se tienen en cuenta la producción de las plantas. Con el propósito de captar las interacciones dinámicas entre diferentes variables que influyen significativamente en la instalación y posterior producción de plantas. A continuación, se procede a definir las variables utilizadas para la construcción de la hipótesis dinámica (Figura 3).

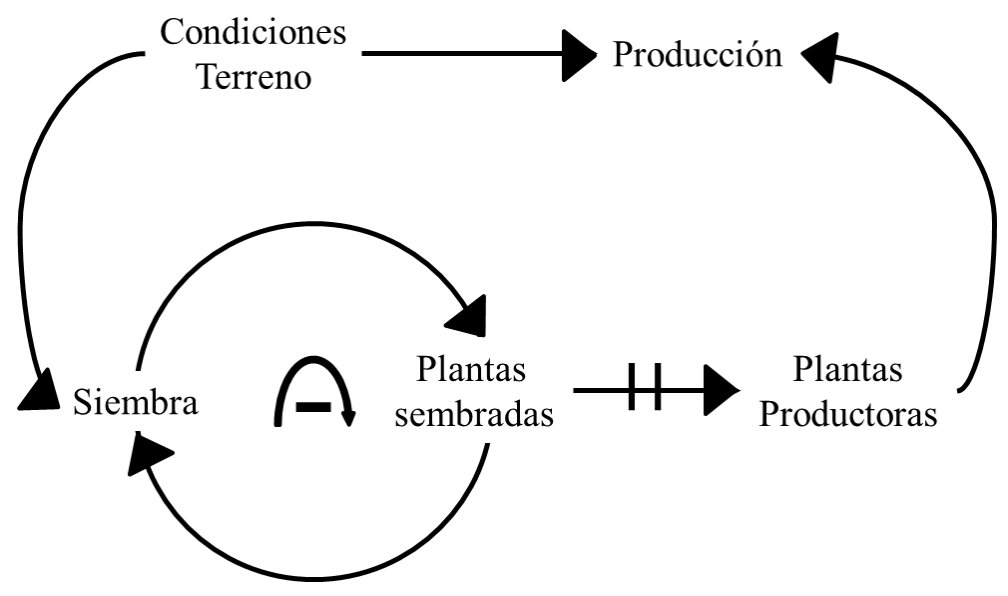

Figura 3. Hipótesis dinámica. 


\subsection{Diagrama de Flujos y Niveles $^{1}$}

El diagrama de Flujo-Nivel se realiza utilizando lógica difusa y lógica clásica para la selección del terreno, con el fin de comparar los resultados del análisis del terreno del modelo en donde se utiliza lógica clásica [18]. Los modelos que aquí se presentan se realizaron en el software Evolución $[2]^{2}$. Los elementos con los cuales cuenta Evolución se presentan en el Cuadro 2.

\begin{tabular}{|c|c|c|}
\hline Elemento & Nombre & Descripción \\
\hline$\theta$ & Parámetro & $\begin{array}{l}\text { Elemento del modelo independiente del sis- } \\
\text { tema o constante propia de este que no varía } \\
\text { durante la simulación. }\end{array}$ \\
\hline$\square$ & Nivel & $\begin{array}{l}\text { Es una variable de estado. Representa la } \\
\text { acumulación de flujos. }\end{array}$ \\
\hline 呫 & Flujo & $\begin{array}{l}\text { Es la variación de un nivel. Representa un } \\
\text { cambio en el estado del sistema. }\end{array}$ \\
\hline$\infty$ & FIS & $\begin{array}{l}\text { Permite definir una variable en términos de } \\
\text { un sistema de inferencia difuso. }\end{array}$ \\
\hline 回 & Retardo & $\begin{array}{l}\text { Elemento que simula retrasos en la transmi- } \\
\text { sión de la información o de material entre } \\
\text { los elementos del sistema. }\end{array}$ \\
\hline$\square$ & Tabla & $\begin{array}{l}\text { Representa una relación de no linealidad } \\
\text { entre dos variables. }\end{array}$ \\
\hline & Auxiliar & $\begin{array}{l}\text { Cantidad con cierto significado para el mo- } \\
\text { delador y con un tiempo de respuesta inme- } \\
\text { diato. }\end{array}$ \\
\hline (อ) & Exógena & $\begin{array}{l}\text { Variable cuya evolución es independiente de } \\
\text { las del resto del sistema. Representa una } \\
\text { acción del medio sobre el sistema. }\end{array}$ \\
\hline 8 & Canal de información & $\begin{array}{l}\text { Es la transmisión de información que no } \\
\text { necesita conservarse. }\end{array}$ \\
\hline
\end{tabular}

Cuadro 2. Elementos de Evaluación

El modelo tiene dos sectores el "crecimiento plantas" y "Factores de clima y exigencias de suelo", la Figura 4 presenta el diagrama de Flujo - Nivel del

${ }^{1}$ Los modelos de lógica difusa y lógica clásica se encuentran disponibles en: www.dropbox.com/sh/vtjbihy23te3nkn/AAD79x1iE5yh5PdHaic0JPS3a?dl=0

${ }^{2}$ Herramienta para el modelado y simulación con Dinámica de Sistemas software, desarrollado por grupo SIMON de Investigación en Modelado y Simulación de la Universidad Industrial de Santander. Disponible para descargar en: http://simon.uis.edu.co 
crecimiento de las plantas teniendo en cuenta la selección de suelos para su instalación, en este modelo se utilizan controladores basados en lógica difusa y usando lógica clásica, para determinar la calidad del terreno, teniendo en cuenta las condiciones climáticas y las exigencias del suelo.
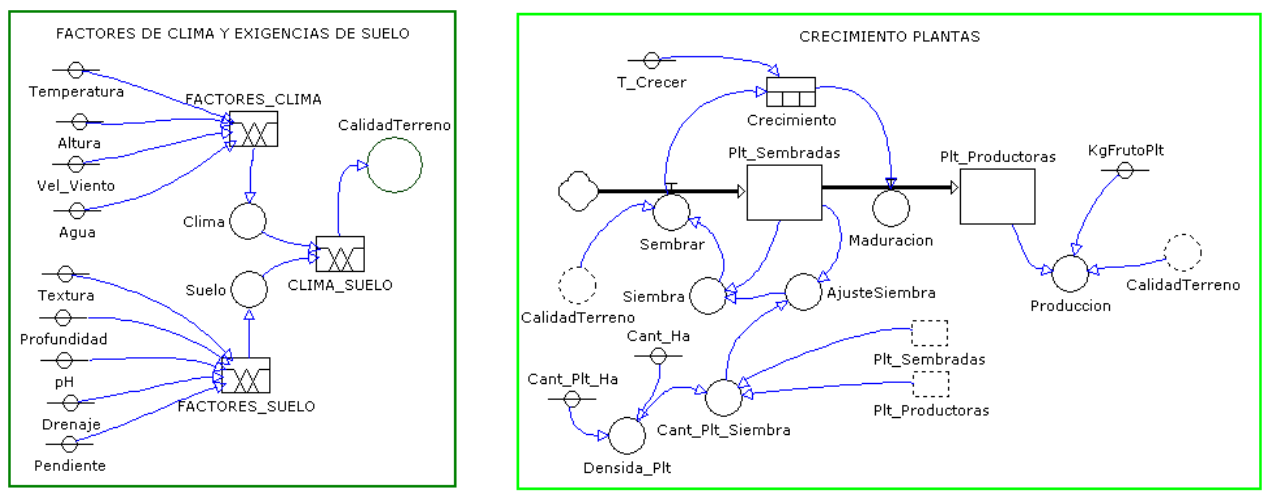

Figura 4. Modelo del crecimiento de plantas.

Para evaluar la calidad del terreno, teniendo en cuenta las los factores climáticos y las exigencias del suelo para un determinado cultivo, se debe diligenciar el Cuadro 3, en donde se presentan los parámetros técnicos principales de un cultivo, estos datos se pueden obtener directamente de los expertos o desde fuentes especializadas. Para este caso se tiene los parámetros técnicos del cultivo de cacao.

La selección de suelos se realiza utilizando lógica difusa y lógica clásica, con el fin de comparar y observar los resultados.

\subsection{Diagrama de Flujos y Niveles usando lógica difusa y lógica clásica}

Construcción del controlador difuso para la selección de suelos de cultivos agrícolas. Para determinar la calidad del terreno para un cultivo específico el experto en agroindustria debe considerar integralmente los diferentes factores del clima y las exigencias del suelo sean óptimas. En este sector se encuentran las variables que permiten indicar la calidad del terreno; con respecto a los factores climáticos se analizan la temperatura, altura, velocidad del viento y precipitación anual, y con respecto a las exigencias del suelo se analizan la textura, profundidad, pH, drenaje y pendiente. Con los resultados de estas dos variables se obtiene la calidad del terreno [12]. Para determinar qué tan apropiado es el terreno se dispone de la herramienta de lógica difusa. Por medio del componente FIS (lógica difusa) de Evolución, se obtienen resultados parecidos a los del razonamiento humano.

A continuación se presenta el ejemplo de la creación de los conjuntos difusos para la variable Temperatura, utilizando el componente FIS. 


\section{FACTORES CLIMÁTICOS}

\begin{tabular}{|c|c|c|c|}
\hline Parámetros Técnico & $\begin{array}{l}\text { Condiciones } \\
\text { Óptimas }\end{array}$ & $\begin{array}{l}\text { Condiciones } \\
\text { Intermedias }\end{array}$ & $\begin{array}{l}\text { Sin Poten- } \\
\text { cial }\end{array}$ \\
\hline \multirow{2}{*}{ Temperatura promedio anual ${ }^{\circ} \mathrm{C}$} & \multirow{2}{*}{$23-25$} & $19-23$ & $<19$ \\
\hline & & $25-32$ & $>32$ \\
\hline Precipitación anual (mm) & $1500-2500$ & $25-32$ & $>32$ \\
\hline Altitud (m.s.n.m) & $400-900$ & $\begin{array}{l}0-400 \\
900-1200\end{array}$ & $>1200$ \\
\hline $\begin{array}{llll}\text { Velocidad máxima del viento } \\
(\mathrm{Km} / \mathrm{H})\end{array}$ & $<10$ & 10 a 14 & $>14$ \\
\hline \multicolumn{4}{|c|}{ SUELOS } \\
\hline Textura & $\begin{array}{l}\text { Franco- } \\
\text { Arcillosa }\end{array}$ & $\begin{array}{l}\text { Franco- } \\
\text { Arenosa }\end{array}$ & $\begin{array}{l}\text { Arenosos- } \\
\text { Arcillosos }\end{array}$ \\
\hline Profundidad (m) & 1.5 & $1.2-1.5$ & $<1.2$ \\
\hline \multirow{2}{*}{$\mathrm{pH}$} & \multirow{2}{*}{$6.0-6.5$} & $5.0-6.0$ & $<5.0$ \\
\hline & & $6.5-7.5$ & $>7.5$ \\
\hline Drenaje & Bueno & Moderado & Deficiente \\
\hline Pendiente & $0-9$ & $9-12$ & $>13$ \\
\hline
\end{tabular}

Cuadro 3. Parámetros técnicos. Fuente: [5]

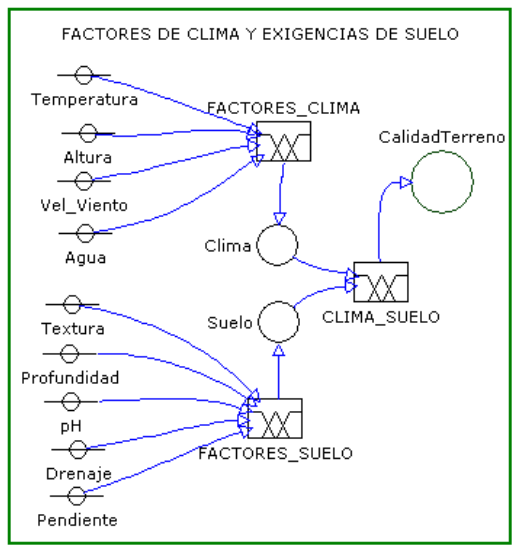

Figura 5. Controlador para selección del suelo usando lógica difusa.

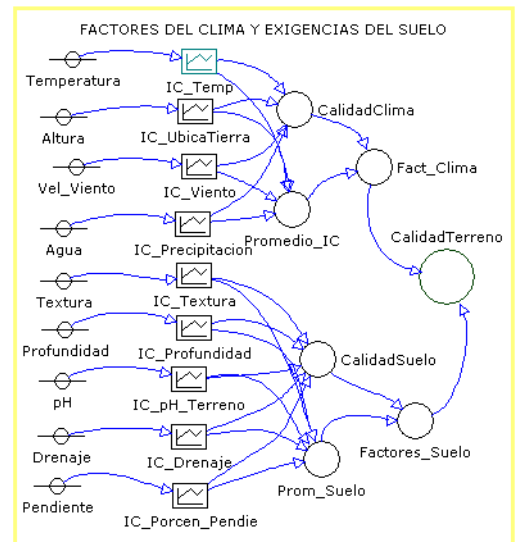

Figura 6. Controlador para la selección de suelo usando lógica clásica.

Este proceso, se debe realizar para cada uno de los parámetros técnicos, de esta forma se asegura que al introducir los datos de un terreno en el modelo, estos determinaran las condiciones del terreno. 
Identificar los parámetros técnicos del cultivo

1

\begin{tabular}{|c|c|c|c|}
\hline Parámetros Técnicos & Condiciones Óptimas & Condiciones Intermedias & Sin Potencial \\
\hline Temperatura Promedio Anual ${ }^{\circ} \mathrm{C}$ & $23-25$ & $19-23$ & $<19$ \\
& & $25-32$ & $>32$ \\
\hline
\end{tabular}

Realizar los conjuntos difusos en el componente FIS de Evolución [17]

2

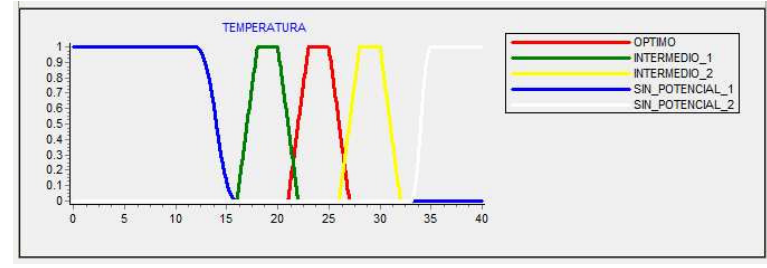

De acuerdo con la información del cuadro 4 se deben crear cinco conjuntos difusos. Después que se han creado todos los conjuntos difusos asociados, se procede a crear las reglas en donde se relacionan las variables.

Cuadro 4. Ejemplo de la creación de los conjuntos difusos de los parámetros técnicos

\subsection{Ecuaciones}

Formulada la hipótesis dinámica que soporta la construcción del modelo dinámico sistémico, en la Figura 4 se presenta el Diagrama de Flujos y Niveles, y sus respectivas ecuaciones matemáticas pueden ser consultadas en el Cuadro 5, el Cuadro 6 y el Cuadro 7.

\begin{tabular}{|c|c|c|c|}
\hline Tipo de variable & Nombre de la variable & Unidades & Ecuación valor inicial \\
\hline \multirow{2}{*}{ Nivel } & Plt_Sembradas & Plantas & 0 \\
\hline & Plt_Productoras & Plantas & 0 \\
\hline \multirow{2}{*}{ Flujo } & Sembrar & Plantas/t & INT(IF(Terreno $>40$, Siembra,0) \\
\hline & Maduracion & Plantas/t & INT(Crecimiento) \\
\hline \multirow{5}{*}{ Auxiliar } & Densidad_Plt & Plantas & Cant_Ha*Cant_Plt_Ha \\
\hline & Cant_Plt_Siembra & Plantas & $\begin{array}{l}\text { IF }(\text { Densida_Plt }=(\text { Plt_Sembradas }+ \text { Plt_Productoras }), 0, \\
\text { Densida_Plt- }(\text { Plt_Sembradas }+ \text { Plt_Productoras })\end{array}$ \\
\hline & Siembra & Plantas & IF(Densida_Plt $<=$ Plt_Sembradas, 0, AjusteSiembra) \\
\hline & AjusteSiembra & Plantas & $\begin{array}{l}\text { IF }((\text { Cant_Plt_Siembra-Plt_Sembradas })>0, \\
(\text { Cant_Plt_Siembra-Plt_Sembradas }), 0)\end{array}$ \\
\hline & Producción & $\mathrm{Kg}$ & (Plt_Productoras*KgFrutoPlt)*[(Terreno)/100] \\
\hline \multirow{4}{*}{ Parámetro } & Cant_Plt_Ha & Plantas/Ha & 1000 \\
\hline & Cant_Ha & $\mathrm{Ha}$ & 1 \\
\hline & T_Crecer & $\mathrm{t}$ & 60 \\
\hline & Kg_Fruto_Plt & $\mathrm{Kg}$ & 5 \\
\hline$\overline{R e t a r d o}$ & Crecimiento & $\mathrm{t}$ & RETARDO(Sembrar, T_Crecer, T_Crecer-12, 0) \\
\hline
\end{tabular}

Cuadro 5. Ecuaciones matemáticas para Diagrama de Flujos y Niveles 


\begin{tabular}{|c|c|c|c|c|c|}
\hline \multicolumn{3}{|c|}{ Tipo de variable Nombre de la variable Unidades } & \multicolumn{3}{|c|}{ Ecuación Valor-Inicial } \\
\hline \multirow{3}{*}{ Auxiliar } & Terreno & \multicolumn{4}{|c|}{ Adimensional CLIMA_SUELO_NUEVA_SALIDA_1 } \\
\hline & Clima & \multicolumn{4}{|c|}{ Adimensional FACTORES_CLIMA_NUEVA_SALIDA_1 } \\
\hline & Suelo & \multicolumn{4}{|c|}{ Adimensional FACTORES_SUELO_NUEVA_SALIDA_1 } \\
\hline \multirow{9}{*}{ Parámetro } & Temperatura & ${ }^{\circ} \mathrm{C}$ & \multicolumn{3}{|l|}{25} \\
\hline & Altura & m.s.n.m & \multicolumn{3}{|l|}{100} \\
\hline & Vel_Viento & $\mathrm{Km} / \mathrm{H}$ & \multicolumn{3}{|l|}{6} \\
\hline & Agua & $\mathrm{mm}$ & \multicolumn{3}{|l|}{2000} \\
\hline & Textura & $\%$ & \multicolumn{3}{|l|}{95} \\
\hline & Profundidad & $\mathrm{m}$ & \multicolumn{3}{|l|}{5} \\
\hline & $\mathrm{pH}$ & \multicolumn{4}{|c|}{ Adimensional 6} \\
\hline & Drenaje & $\%$ & \multicolumn{3}{|l|}{95} \\
\hline & Pendiente & $\%$ & \multicolumn{3}{|l|}{1} \\
\hline \multirow{34}{*}{ FIS } & \multirow{34}{*}{ FACTORES_CLIMA } & \multirow{34}{*}{ Adimensiona } & \multicolumn{3}{|c|}{ Entradas } \\
\hline & & & \multicolumn{3}{|l|}{ - Temperatura } \\
\hline & & & \multicolumn{3}{|c|}{ Universo de discurso: $[0,40]$} \\
\hline & & & \multicolumn{2}{|c|}{ Nombre Conjunto Parámetros } & Función Membresía \\
\hline & & & Óptimo & {$[21,23,25,27]$} & Trapezoidal \\
\hline & & & Intermedio 1 & {$[16,18,20,22]$} & Trapezoidal \\
\hline & & & Intermedio 2 & {$[26,28,30,32]$} & Trapezoidal \\
\hline & & & Sin potencial 1 & {$[12,16]$} & Tipo Z \\
\hline & & & Sin potencial 2 & {$[33,35]$} & Tipo S \\
\hline & & & - Altura & & \\
\hline & & & Universo de discur & rso: $[0,1500]$ & \\
\hline & & & Nombre conjunto & Parámetros & Función de membresía \\
\hline & & & Óptimo & {$[300,500,1100,1300]$} & Trapezoidal \\
\hline & & & Intermedio & {$[250,300,400,450]$} & Trapezoidal \\
\hline & & & $\overline{\text { Sin potencial }}$ & {$[1000,1500]$} & Tipo S \\
\hline & & & - Vel_Viento & & \\
\hline & & & Universo de discur & rso: $[0,20]$ & \\
\hline & & & Nombre conjunto & Parámetros & Función de membresía \\
\hline & & & Óptimo & {$[-1,0,10,11]$} & Trapezoidal \\
\hline & & & Intermedio & {$[10,12,14]$} & Triangular \\
\hline & & & Sin potencial & {$[14,20]$} & Tipo S \\
\hline & & & - Agua & & \\
\hline & & & Universo de discur & rso: $[0,2600]$ & \\
\hline & & & Nombre conjunto & Parámetros & Función de membresía \\
\hline & & & Óptimo & {$[1200,1300,2500,2600$} & Trapezoidal \\
\hline & & & Intermedio & {$[900,1000,1100,1300]$} & Trapezoidal \\
\hline & & & Sin potencial & {$[-100,0,800,1000]$} & Trapezoidal \\
\hline & & & & Salidas & \\
\hline & & & - NUEVA_SALI & DA_1 & \\
\hline & & & Universo de discur & rso: $[0,100]$ & \\
\hline & & & Nombre conjunto & Parámetros & Función de membresía \\
\hline & & & Óptimo & {$[75,85,100,105]$} & Trapezoidal \\
\hline & & & Intermedio & {$[45,55,75,85]$} & Trapezoidal \\
\hline & & & Sin potencial & {$[-15,-5,40,50]$} & Trapezoidal \\
\hline
\end{tabular}

Cuadro 6. Ecuaciones matemáticas para el Diagrama de Flujos y Niveles del controlador difuso

El modelo desarrollado aplicando Dinámica de Sistemas se ha diseñado con el propósito de ofrecer un espacio para la exploración y el aprendizaje acerca del crecimiento y producción de las plantas teniendo en cuenta las condiciones del terreno. 


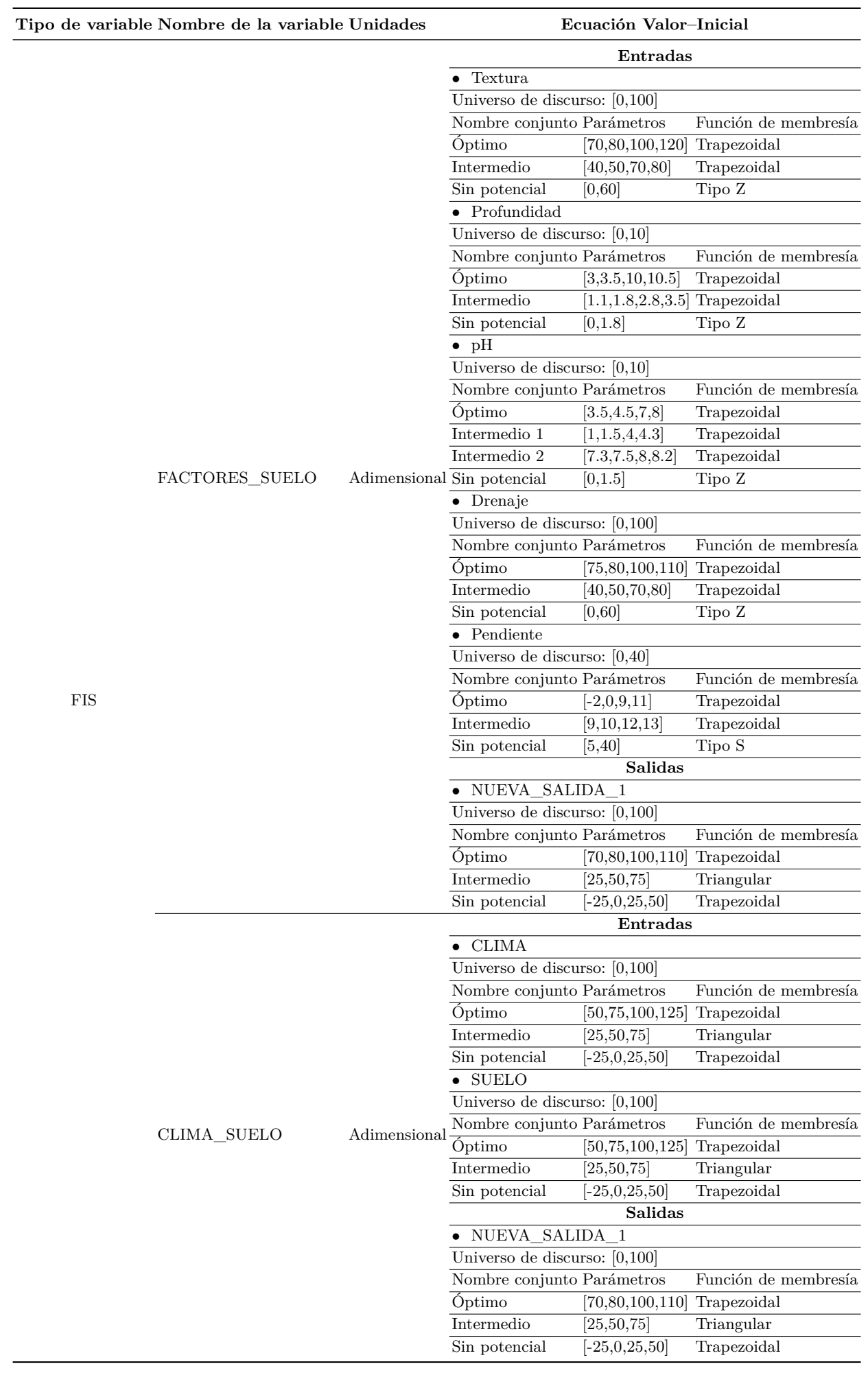

Cuadro 7. Ecuaciones matemáticas para el Diagrama de Flujos y Niveles del controlador difuso 


\begin{tabular}{|c|c|c|c|}
\hline Tipo de variabl & Nombre de la variable & e Unidades & Ecuación valor inicial \\
\hline \multirow{7}{*}{ Auxiliar } & Calidad_Clima & Adimensional & l IC_Temp*IC_UbicaTierra*IC_Viento*IC_Precipitacion \\
\hline & Promedio_IC & Adimensional & l (IC_Temp+IC_UbicaTierra+IC_Viento+IC_Precipitacion) $/ 4$ \\
\hline & Fact_Clima & Adimensional & Il IF(CalidadClima $<>0$,Promedio_IC,0) \\
\hline & Calidad_Suelo & Adimensional & $\begin{array}{l}\text { IC_Profundidad*IC_pH_Terreno*IC_Drenaje*IC_Porcen_Pendie } \\
{ }^{*} \text { IC_Textura }\end{array}$ \\
\hline & Prom_Suelo & Adimensional & $\begin{array}{l}\text { (IC_Profundidad+IC_pH_Terreno+IC_Drenaje+IC_Porcen_Pendie } \\
\left.+\mathrm{IC} \_ \text {Textura }\right) / 5\end{array}$ \\
\hline & Factores_Suelo & Adimensional & IF (CalidadSuelo==0,0,Prom_Suelo) \\
\hline & Terreno & Adimensional & l(Fact_Clima+Factores_Suelo) $/ 2$ \\
\hline \multirow{9}{*}{ Parámetro } & Temperatura & ${ }^{\circ} \mathrm{C}$ & 25 \\
\hline & Altura & m.s.n.m & 100 \\
\hline & Vel_Viento & $\mathrm{Km} / \mathrm{H}$ & 6 \\
\hline & Agua & $\mathrm{mm}$ & 2000 \\
\hline & Textura & $\%$ & 95 \\
\hline & Profundidad & $\mathrm{m}$ & 5 \\
\hline & $\mathrm{pH}$ & Adimensional & 6 \\
\hline & Drenaje & $\% 1$ & 95 \\
\hline & Pendiente & $\%$ & 1 \\
\hline \multirow{9}{*}{ Tabla } & IC_Temp & Adimensional & $\begin{array}{l}\text { INTLINEAL }(0,0,1,0,0,0,0,0,0,0,0,0,0,0,0,0,0,0,0,0,0,0,0.8,0.9,0.95,1,1,1,1 \text {, } \\
0.9,0.9,0.9,0.9,0.8,0.7,0,0,0,0,0,0,0,0,0,0,0,0,0,0)\end{array}$ \\
\hline & IC_UbicaTierra & Adimensional & Il INTLINEAL $(0,0,100,0.5,0.6,0.7,0.8,0.9,1,1,1,1,1,0.9,0.8,0.7,0,0,0)$ \\
\hline & IC_Viento & Adimensional & l INTLINEAL $(0,0,1,1,1,1,1,1,1,1,1,1,1,1,0.9,0.8,0.7,0.6,0,0,0,0,0,0,0)$ \\
\hline & IC_Precipitacion & Adimensional & $\begin{array}{l}\text { INTLINEAL }(0,0,100,0,0,0,0,0,0,0,0,0,0,0.6,0.7,0.8,0.9,1,1,1,1,1,1,1,1,1,1 \text {, } \\
1,1,0.7)\end{array}$ \\
\hline & IC_Textura & Adimensional & liNTLINEAL $(0,0,10,0,0,0,0.5,0.6,0.7,0.8,0.9,1,1,1)$ \\
\hline & IC_Profundidad & Adimensional & $\begin{array}{l}\text { INTLINEAL }(1,0,0.3,0,0,0,0,0,0,0.6,0.7,0.8,0.9,1,1,1,1,1,1,1,1,1,1,1,1,1,1, \\
1,1,1,1,1,1,1,1,1,1,1,1)\end{array}$ \\
\hline & IC_pH_Terreno & Adimensional & $\begin{array}{l}\text { INTLINEAL }(0,0,0.5,0,0,0,0.7,0.75,0.8,0.85,0.9,0.95,1,1,1,1,1,1,0.9,0.7 \\
0.5)\end{array}$ \\
\hline & IC_Drenaje & Adimensional & I INTLINEAL $(0,0,10,0,0,0,0,0.6,0.7,0.8,0.9,1,1,1)$ \\
\hline & IC_Porcen_Pendiente & Adimensional & $\begin{array}{l}\text { INTLINEAL }(0,0,1,1,1,1,1,1,1,1,1,1,1,0.9,0.8,0.7,0.6,0.5,0,0,0,0,0,0,0,0,0 \\
0,0,0,0,0,0,0,0,0,0,0,0,0)\end{array}$ \\
\hline
\end{tabular}

Cuadro 8. Ecuaciones matemáticas para el Diagrama de Flujos y Niveles del controlador clásico

\subsection{Comportamientos}

Los resultados se presentan a continuación para una combinación de posibles escenarios, en donde solo se van a modificar los valores de los parámetros asociados al clima y suelo; los demás valores corresponden a los presentados en las cuadros $6,7,8$ y 9 . En el primer escenario se observa que aunque se tiene un terreno que se encuentra con parámetros de condiciones óptimas, la pendiente, se encuentra en un rango sin potencial según la literatura; sin embargo la literatura también nos dice que este parámetro no es determinante en la selección del terreno. Con lógica clásica no se puede pertenecer a dos conjuntos, es por esto que las exigencias del suelo no se cumplen y al promediar solo se tendría en cuenta las condiciones de clima y por esto se obtiene el resultado de $47.5 \%$ de calidad del terreno, y como para iniciar la siembra el terreno debe tener condiciones intermedias altas superior a $70 \%$, no se cumple la condición, luego no hay siembra y por lo tanto tampoco hay producción. Por otra parte, con lógica difusa, como la altura no es determinante para descartar el terreno, esta evalúa los demás parámetros y se obtiene un resultado de $87.5 \%$ y se puede realizar la siembra y obtener producción. 
Sin embargo, al cambiar el valor de la pendiente por un dos, el cual es un valor que se encuentra en rango óptimo, se obtiene que la lógica clásica, considere el terreno óptimo; como se muestra en el segundo escenario.

En el tercer escenario, con condiciones óptimas se observa que aunque los presentan resultados similares, la lógica clásica da una puntuación máxima al terreno con un valor del $100 \%$, mientras que la lógica difusa aunque considera que aunque el terreno es muy bueno nunca llega a calificarlo con un valor del $100 \%$, tal como lo hace una persona, la cual siempre deja un margen de duda.

En el cuarto escenario, se consideran condiciones intermedias de clima y suelo, y mientras que el controlador clásico evalúa el terreno con un $85.7 \%$, el controlador difuso lo evalúa con un $76.7 \%$, en este escenario se debe reflexionar muy bien antes de realizar la instalación del cultivo, ya que aunque tiene condiciones intermedias, el rendimiento del cultivo no es tan favorable con respecto a la inversión que se debe hacer.

Para el escenario 5, en donde se tiene parametros tanto sin potencial, como intermedios y óptimos, los dos controladores nos indican que no es un terreno adecuado para el cultivo de cacao. La agricultura colombiana ha tenido un incremento no solo en los productos tradicionales de exportación como el banano sino también de nuevos exportables como palma, frutas y hortalizas. El crecimiento del mercado interno también ha contribuido a incrementar la producción en el caso del plátano, papa, frutas, entre otras; evidenciando el potencial de desarrollo al interior del país [22].

Como resultado el país ha presentado una producción agrícola creciente y diversificada. Del total de la producción durante el 2009, el $66 \%$ perteneció a cultivos permanentes mientras que el $34 \%$ restante a cultivos transitorios. Sin embargo, solo el $3 \%$ del territorio es usado para fines agrícolas, y el potencial de expansión de tierras del país es alto, "según la $\mathrm{FAO}^{3}$, Colombia se ubica en el puesto 25 entre 223 países en donde se evalúa el potencial de expansión del área agrícola sin afectar el bosque natural. Este potencial de crecimiento según el Ministerio de Agricultura y Desarrollo Rural se estima en 10 millones de hectáreas, dentro de las que se encuentran áreas no aprovechadas y otras que han sido utilizadas sin atender criterios de vocación productiva" [27].

Actualmente, se necesita realizar estudio a los terrenos con el fin de conocer la vocación productiva, y es en ahí, en donde el modelado y simulación, permite a los productores y expertos de la agroindustria, analizar la calidad del terreno para un cultivo determinado por medio de controladores lógicos, con el fin de conocer que tan apropiado es el terreno para determinado cultivo y la relación entre la calidad del terreno y los rendimientos por hectarea.

\section{Conclusiones}

La integración de la Dinámica de Sistemas y la Lógica Difusa constituyen una herramienta útil para abordar el análisis del terreno y como afecta la producción

\footnotetext{
${ }^{3}$ Organización de las Naciones Unidas para la Agricultura y la Alimentación, FAO(Food and Agriculture Organization por sus siglas en Inglés)
} 

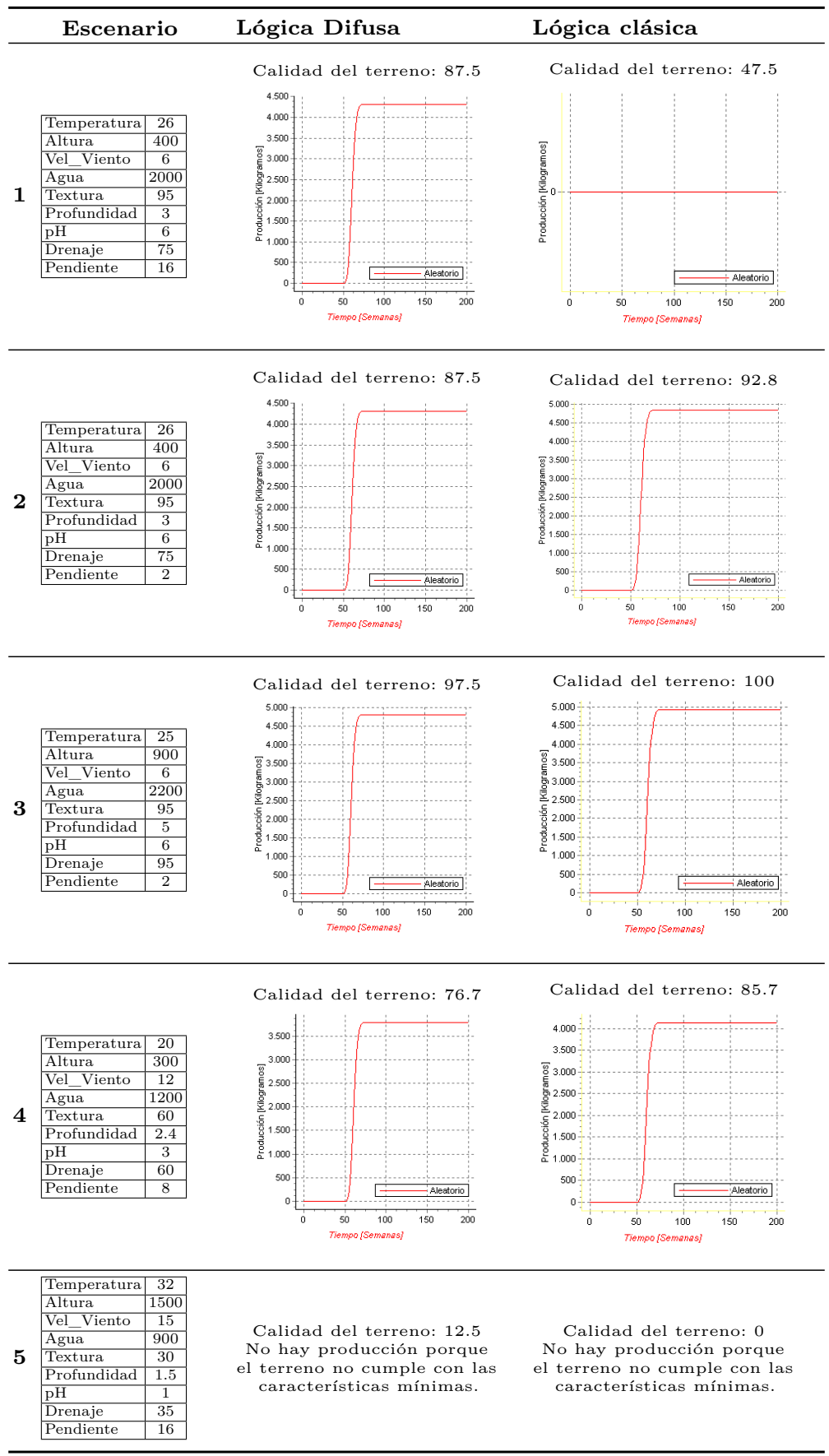

Cuadro 9. Presentación de resultados de simulación. Fuente: Autor

de sistemas agrícolas. Ya que apoya al especialista en producción en la toma de decisiones argumentada a partir de simulaciones realizadas por el modelo dinámico. Lo cual, le permite experimentar de forma iterativa para evaluar posibles escenarios. Esta área ha demostrado ser una aproximación eficiente 
para aplicaciones de ámbito muy variado: industrial, agrícola, medio ambiente, espacial, químico, navegación aérea y marítima, entre otras.

Aprovechando las características de la Lógica Difusa como que soporta datos imprecisos, es fácil de entender, flexible, se basa en el lenguaje humano y experiencia de expertos conocedores del problema; los actores involucrados participan en la construcción del modelo, aportando su conocimiento experto para la creación y posterior validación de las variables difusas que en el modelo representan la selección del terreno para un determinado cultivo.

La aplicación de la Lógica Difusa, permite abordar problemas complejos como es el control de sistemas no lineales. En este caso, se ha aplicado a la selección de suelos para cultivos agrícolas.

Finalmente, el uso de herramientas tecnológicas soportadas en dinámica de sistemas permite incrementar la productividad de estos sistemas productivos. Debido a que el modelo dinámico sistémico que soporta el funcionamiento de la herramienta tecnológica, complementa la racionalidad del productor.

\section{Referencias}

1. Andrade Sosa, H.H. et al., 2010. Evolución: herramienta software para modelado y simulación con Dinámica de Sistemas. Revista de Dinámica de Sistemas.

2. Andrade Sosa, H.H. et al., 2001. Pensamiento Sistémico: Diversidad en búsqueda de unidad. Bucaramanga: Ediciones Universidad Industrial de Santander.

3. Aracil, J., 1995. Dinámica de sistemas. Isdefe., Madrid.

4. Arango Serna, M.D., Adarme Jaimes, W. \& Zapata Cortes, J.A., 2010. Gestión de abastecimiento - Logística con indicadores bajo incertidumbre, caso aplicado sector panificador Palmira.

5. Borrero, C., 2008. El cultivo de cacao en el Guaviare.

6. Brunet Cortina, I. \& Alfonso Robaina, D., 2010. Lógica difusa aplicada a la toma de decisiones. Industrial XXXI.

7. Coyle, R.G., 1996. System Dynamics Modelling: A Practical Approach. Ilustrada,. CRC Press, ed.

8. Danilo, O., Giraldo, M. \& Buitrago, D.G., 2013. Lógica Difusa Aplicada al Control Local del Péndulo Invertido con Rueda de Reacción. , 18(4), pp.623-632.

9. F, O., Gayar, E. \& Leung, P., 2001. A multiple criteria decision making framework for regional aquaculture development. European Journal of Operational Research. 133(3), pp.462-482.

10. F, O., Gayar, E. \& Leung, P., 2000. ADDSS: a tool for regional aquaculture development. Aquacultural Engineering. 23(1), pp. 181-202.

11. Forrester, J., 2013. Industrial Dynamics. M. Publishing, ed.,

12. García Benítez, G. \& Vera Estrada, C., 2010. Un modelo de sistema dinámico híbrido utilizando el enfoque de la lógica difusa. CIENCIA ergo sum., 17(2), pp. $165-175$.

13. Gómez, U.E., Andrade, H.H. \& Vásquez, C.A., Lineamientos Metodológicos para construir Ambientes de Aprendizaje en Sistemas Productivos Agropecuarios soportados en Dinámica de Sistemas. (Spanish). Información Tecnológica. 26(4), pp.125-135. Available at: 10.4067/S0718-07642015000400016.

14. González, C., 2012. Lógica Difusa: Una introducción práctica., p.29. 
15. De la Rosa, D., 2008. Evaluación agro-ecológica de suelos para un desarrollo rural sostenible. Ediciones., Madrid.

16. Londoño Ciro, L.A. et al., Uso de micromundos con Dinámica de Sistemas y lógica difusa para el diseño de evaluación de competencias en ciencias básicas en ingeniería, pp.1-8.

17. Machado Mendoza, G.D., González Pérez, C.E. \& Andrade Sosa, H.H., 2006. Componente de sistema de inferencia difusa (FIS) para Evolución 3.5., Bucaramanga.

18. Martínez Rojas, N., Andrade Sosa, H.H. \& Gomez Prada, U.E., 2011. Propuesta de un modelo con dinámica de sistemas como herramienta en el aprendizaje del eslabón de producción de la cadena productiva del cacao, p.15.

19. Matías, T.B. \& Durán Vicente, M.I., Lógica borrosa.

20. Orji, I.J. \& Wei, S., 2015. An innovative integration of fuzzy-logic and systems dynamics in sustainable supplier selection: A case on manufacturing industry. Computers \& Industrial Engineering., 88, pp. 1-12.

21. Osorio Calderón, P.H., Andrade Sosa, H.H. \& Gómez Prada, U.E., 2010. Propuesta de un modelo de simulación como herramienta en la justificación y comprensión de la toma de decisiones en la inversión pública. Un enfoque sistémico., Bucaramanga.

22. PROEXPORT Colombia, 2014. Análisis de exportaciones colombianas 2013.

23. Santos, M., 2011. Un Enfoque Aplicado del Control Inteligente. Revista Iberoamericana de Automática e Informática Industrial RIAI., 8(4), pp. 283-296.

24. Santos Peña, M. \& Miranda Suescun, E., 2012. Aplicación de la lógica difusa en el ámbito de las energías renovables. Revista Elementos, 2(1), pp. 101-114.

25. Schaffernicht, M., Dinámica de Sistemas: La búsqueda del rigor en el diseño de sistemas sociales.

26. Sterman, J.D., 2000. Business dynamics: Systems thinking and modeling for a complex world., Available at: http://www.lavoisier.fr/notice/frJWOAR6SA23WL00. html.

27. Vélez, A. et al., 2011. Sector Agroindustrial Colombiano., Bogotá, Colombia. 